British Journal of Anaesthetic and Recovery Nursing

http://journals.cambridge.org/ARN

Additional services for British Journal of Anaesthetic and Recovery Nursing:

Email alerts: $\underline{\text { Click here }}$

Subscriptions: Click here

Commercial reprints: $\underline{\text { Click here }}$

Terms of use : $\underline{\text { Click here }}$

\title{
Delegation Dilemma: Accountability is Undermined by a Lack of Role Clarity
}

Linnette King

British Journal of Anaesthetic and Recovery Nursing / Volume 3 / Issue 02 / May 2002, pp 16 - 19 DOI: 10.1017/S1742645600000929, Published online: 25 February 2009

Link to this article: http://journals.cambridge.org/abstract_S1742645600000929

How to cite this article:

Linnette King (2002). Delegation Dilemma: Accountability is Undermined by a Lack of Role Clarity. British Journal of Anaesthetic and

Recovery Nursing, 3, pp 16-19 doi:10.1017/S1742645600000929

Request Permissions : $\underline{\text { Click here }}$ 


\section{DELEGATION DILEMMA: ACCOUNTABILITY IS UNDERMINED BY A LACK OF ROLE CLARITY}

\section{A BST RACT}

A lack of clarity in role definition can pose a risk to the care of patients in the peri-operative phase. Health care teams are often made up of multi-skilled-nursing-care-providers that are not statutorily registered professionals. This may undermine who is actually responsible and accountable in practice. This delegation dilemma is due to certain market forces, like changes and reforms in health service provision, shortages of doctors and nurses, and ever rising costs. Change and development in practitioners' scope of practice has been encouraged with training to enable practitioners to take on certain specific aspects of health care provision that were traditionally part of a doctor and, or a nurse's role. These changes should be monitored and considered by risk managers or those responsible for clinical governance, as they raise important legal and professional issues. In short, there is confusion over accountability driven by issues of role expansion. The respective professional bodies, both medical and nursing and the Department of Health need to come together and find a consensus of opinion in resolving the confusion over accountability and role delineation.

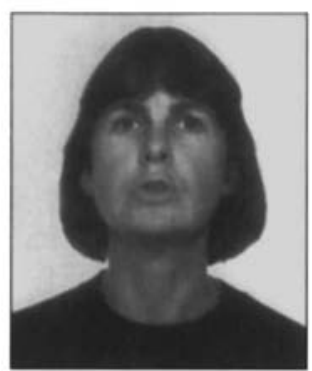

Linnette King RGN, RNT, LLM (LAMP), MSc Nursing, PGDiploma Education, BSc (Hons) Nursing Studies
Role blurring of staff in the perioperative phase of patient care has led to confusion and a lack of understanding over accountability. Accountability is a multifaceted concept (Otto 1999, see figure 1) and complicated by being intertwined with responsibility. The lack of clarity in role definition and responsibility can lead to diminished patient safety due to a decreased continuity of care, and patient centred care because of unclear working practices. These issues also can increase risks and potential for error for patients, raise departmental costs and add to staff dissatisfaction, which in turn is evidenced by a continued recruitment crisis in these acute care areas.

Certain market forces over the years have led to change and reform in health care provision, with the health services appearing to be in a period of continuous review and subsequent change. The particular market forces of interest here are with nurse (and other registered professionals) shortages, adjacent to rising costs of health care provision and, of course, societal expectation. These have been countered by some extent with reforms in the health service structure, for example, the NHS Plan (DOH 2000).

More specifically these changes have driven a skill mix review of health care teams, which have consequently led to multiskilled-nursing-care-providers who are not registered professionals, for example, various types of assistants, technicians, auxiliaries and practitioners. However, the teams must continue to provide quality health care consistent with the standard of care expected by the regulated professional body and by the patient need. All of this requires education, training and skill development, of the respective staff, to ensure patient health and safety is maintained.

Nurses have been encouraged to expand and develop their scope of practice with many encompassing what was traditionally part of the doctor's role. Other care providers have also been trained to take on certain specific aspects of health care provision, particularly where there have been, or continue to be specific shortages of regulated staff, that is, in specialist acute care areas like the operating department. Consequently difficulties have arisen in clarifying role boundaries between these various practitioners (Dowling et al 1996, Tingle 1997). These changes raise important legal and professional issues, which should be a source for consideration by managers of risk, for responsible governance and when a case of clinical negligence arises out of issues from these expanding and diversifying roles.

In short there is an accountability confusion driven by issues of role expansion. Registered professionals must ensure appropriate delegation in their duty of care (see figure 2) which is supported by an expected standard of care ${ }^{1}$. There is some conflicting advice from the regulatory bodies

'Bolam v Friern Hospital Management Committee [1957] 1 WLR 582, states that a competent standard of care is that expected of reasonably skilled and competent practitioner, and that it is sufficient to exercise an ordinary level of skill. Wilsher v Essex AHA [1988] 2 WLR 557, states that the level of competence expected is what is needed for the job, not what the practitioner can offer. Also it made clear that specialist practitioners are expected to have greater skills and competence. 


\section{A Multi-faceted Concept of Accountability} Accountability has many facets and interpretations, but can be categorised as legal, moral and professional. Accountability cannot be delegated as professional practitioners must answer for the care they give and also for the care that they may ask others to do.

Practitioners need to have the ability to be accountable, that is, the willingness or commitment. Accountability involves skills of anticipation, which arise from a systematic process of care through assessment, planning, implementation and evaluation. This will require adequate knowledge and competence through continuous professional growth, development of a professional attitude and integrity in practice. Standards of care must be ensured through regular audit to ensure that the outcomes are met and that they can be measured with appropriate criteria. Management of the consequences of action, non-action, omission and, or delegation have to be taken responsibly. The practitioner is answerable in balancing risks with benefits for patients. Thus protection of the health, safety and welfare of patients should be protected.

Legal accountability is the extent to which a practitioner can be held in law to be liable for their actions (Dimond 1995). Moral accountability has similarity, but a different emphasis and authority, which may lead to moral dilemmas for practitioners. The main ethical principles of autonomy, beneficence, non-maleficence and justice can give rise to difficulties in clinical decision making. Professional accountability is usually defined to some extent by the professional codes and guidelines. Legally and morally the professional practitioner has a duty of care. The public place their trust in a relationship, which arises from the integrity of the communication between patient and practitioner and from practitioner to practitioner. Key notions of confidentiality, informed consent and advocacy are an expectation.

The four arenas of accountability (Dimond 1995) are important to understand:

- from the aspect of the patient and society, which are guided by civil and criminal law;

- to the arena of employment defined by contract, guidelines and policies;

- to the professional body regulations, which set out the standards expected of the registered professional;

- to that of the individual defined by their knowledge, competence and attitude. The latter arising from their personal values and beliefs.

Professional accountability means that the practitioner can be relied upon to carry out what is expected by the public, your employer, your colleagues, your peers and yourself.

Reference: Dimond B [1995] Legal Aspects of Nursing. 2nd Edition London: Prentice Hall

Figure 1 for nurses and doctors respectively. This can be seen when reading the United Kingdom Central Council's Code of Professional Conduct (1992a) and Scope of Professional Practice (1992b) along with the General Medical Council's Duties of a Doctor, Good Medical Practice (1995) and the British Medical Association's Protecting Patient Safety (1996). This is then further confused by current government advice arising from their various proposals and guidance on modernising the NHS, like the NHS Plan (DOH 2000).

The GMC and the BMA guidelines clearly state that doctors are generally accountable for all care, including that given by other practitioners (GMC 1995, BMA 1996) as per the following two extracts.

'You may delegate medical care to nurses and other healthcare staff who are not registered medical practitioners if you believe it is best for the patient. But you must be sure that the person to whom you delegate is competent to undertake the procedure or therapy involved. When delegating care or treatment, you must always pass on enough information about the patient and the treatment needed. You will still be responsible for managing the patient's care" (GMC 1995, clause 28).

'You must not enable anyone who is not registered with the GMC to carry out tasks that require the knowledge and skills of a doctor" (GMC 1995, clause 29).

These two clauses should be read together otherwise clause 29 would preclude any other practitioner expanding their role. Clause 28 places a direct obligation on the doctor and qualifies clause 29 by stating that a competent nurse or other health care staff must assure delegation of care. Although the GMC view the doctor as the overseer of clinical responsibility for the nurse's interventions the respective professionals remain individually, professionally and legally accountable for their actions, omissions and further delegations (Tingle 1997).

The BMA's (1996) guidance echoes that of the GMC viewing the doctor as the delegater and manager of patient care.

"Delegated tasks vary from simple common day-to-day procedures to significant therapeutic interventions. Whatever the level of complexity of 


\section{Effective Delegation}

Delegation is a necessary aspect of the complexity of health care provision. The health service is a large hierarchical organisation, which further necessitates delegation in order to manage the multiple aspects of the service provision to the public. Many practitioners have faced delegation dilemmas in today's changing and pressurised health practice environment. Practitioners now have more diverse roles and responsibilities and patients to care for than previous generations. Five rights of delegation help in managing this process effectively (Fisher 1999):

- Right task - to delegate only those tasks deemed appropriate rather than that which falls within the practitioner's scope of practice alone;

- Right circumstances - to consider the environment including such criteria as the staffing numbers, mix and needs together with the patient'(s) needs and other resources that may be required;

- Right person - to ensure that the practitioner is adequately qualified and competent to manage the task. Policy, standards and guidelines should enable the process;

- Right communication - to provide clear, concise description of the task, which includes the objective, qualification, competency and expectation;

- Right feedback - to provide appropriate supervision, adequate time and resources, and reasonable evaluation of the practitioner's activity to ensure a reasonable standard of intervention.

Effective delegation means that the practitioner must bear the responsibility of any action or inaction for themselves and of those to whom they have delegated tasks to. If the practitioner understands the principles of delegation and implements them responsibly then appropriate delegation should occur. The nursing process is a conceptual model that may facilitate effective delegation through appropriate assessment, sound planning, competent implementation, supportive supervision and measurable evaluation. It is the implementation stage that is delegated whilst the other facets of this process are the remit of professional expertise. Professional regulations, employers job descriptions, policies and standards, along side the legislative measures should all provide guidance for effective delegation.

Reference: Fisher M [1999] Do your nurses delegate effectively? Nursing Management 30 [5] $23-6$

Figure 1 the task, the doctor responsible for delegating the task and the healthcare professional responsible for performing the task should always take account of a number of issues...

The name of the general practitioner or hospital consultant holding ultimate clinical responsibility should be known to the patient and all members of the healthcare team. The doctor will be responsible for any decision to delegate responsibility.

The healthcare professional to whom the clinical task is to be delegated must have received the appropriate training to be able to perform the task. He/she must be able to recognise complications and know whom to report should problems occur.

Protocols describing and limiting the task to be performed must be produced. The degree of supervision should be described within the protocol. There should be regular audit of task performance and outcome.

The status of the person performing the procedure must be clearly apparent to the patient; the patient's consent must be obtained before a non-medically qualified healthcare professional performs a clinical task that has been delegated by a doctor. There will be circumstances where the delegation of clinical tasks to non-medically qualified staff is clearly inappropriate" (BMA 1996).

The Scope of Professional Practice guidelines (UKCC 1992b) views the expanded role of a nurse from a different stance. This guidance focuses on the individual personal accountability of the nurse and directs nurses in their practice to the guiding principles set out in the Code of Professional Conduct (UKCC 1992a) and the principles set out in clauses eight to ten of the Scope guidelines. No direct crossreference is made to the doctors overall responsibility in either document leaving nurses blithely unaware of the GMC or BMA statements. Thus, as there is no consensus between these regulated professions the uncertainty will remain. This confusion is further illustrated by research (Dowling et al 1996), which is further emphasised by 
conflicting decisions made in the courts (Tingle 1997). When all aspects are compared the regulations from the professional bodies are contradictory and in some respects difficult to apply to actual working practices.

There is a strong case for asking the professional bodies and the Department of Health to come together and find a consensus of opinion in resolving the confusion over accountability and role delineation. Currently the Nursing and Midwifery Council is taking over from the UKCC and one of its primary tasks will be to review the Code of Professional Conduct, Scope of Professional Practice and other nursing guidelines. There has been much consultation and research leading up to this review (UKCC 2001), but the test will be in whether a new ethical code and set of guidelines will help to clarify for nurses and other practitioners their position in the proliferation of roles that are now seen. Also, just as important is that the GMC with the BMA will need to respond by revising their code and guidelines correspondingly. The Department of Health needs to provide clearer guidance to health service managers, and health service managers should ensure that staff job descriptions, roles and responsibilities are clear and regularly reviewed.

\section{References}

British Medical Association [1996] Protecting Patient Safety: Medical Procedures Performed by Non-Medically Qualified Health Professionals Joint Consultants' Committee February BMA: London

Dimond B [1995] Legal Aspects of Nursing. 2nd Edition London: Prentice Hall

Dowling $S$ et al [1996] Nurses taking on junior doctors' work: a confusion of accountability British Medical Journal 31 [2] 12211 - 4

Fisher M [1999] Do your nurses' delegate effectively? Nursing Management 30 [5] 23 - 6

General Medical Council [1995] Duties of a Doctor, Good Medical Practice October GMC: London.

The NHS Plan [2000]. www.nhs.uk/nationalplan

Otto D [1999] Regulatory statutes and issues clinical accountability in perioperative settings AORN Journal 70 [2] 241 - 251

Tingle $J$ [1997] Expanded role of the nurse: accountability confusion British Journal of Nursing 6 [17] 1010 - 3

United Kingdom Central Council for Nurses, Midwives and Health Visitors [1992a] Code of Professional Conduct. London: UKCC

United Kingdom Central Council for Nurses, Midwives and Health Visitors [1992b] The Scope of Professional Practice London: UKCC

United Kingdom Central Council for Nurses, Midwives and Health Visitors [2001] Register Autumn number 37

\section{STANDARDS - STANDARDS - STANDARDS}

The initial meeting to discuss standards in anaesthetics and recovery took place on 5th April 2002 at St George's Hospital. Unfortunately it took place after the deadline for this is of the journal. However there will be a full report in the August issue.

The meeting was led by BARNA chair Pat Smedley who got together several people who work in anaesthetics and recovery who are not BARNA committee members to give an new perspective to standards. It is hoped to develop some nationally recognised standards applicable to anaesthetics and recovery.

We would like to hear from you. Perhaps you could feed this through your link member. If you have any examples of standards or you would like contribute something then we would love to hear from you. You can contact Pat by email at patsmedley@hotmail.com or by writing the editor of the journal [address on the back cover of this issue.]

Now BARNA has more time to concentrate on things such as this and we would really appreciate your input. We look forward to hearing from you. 